The incidence of cardiogenic shock in community studies has not decreased significantly over time. Despite decreasing mortality rates associated with increasing utilisation of revascularisation, shock remains the leading cause of death for patients hospitalised with acute myocardial infarction (MI). Although shock often develops early after MI onset, it is typically not diagnosed on hospital presentation. Failure to recognise early haemodynamic compromise and the increased early use of hypotension inducing treatments may explain this observation.

Recently, a randomised trial has demonstrated that early revascularisation reduces six and 12 month mortality. ${ }^{2}$ The current American College of Cardiology/American Heart Association (ACC/AHA) guidelines recommend the adoption of an early revascularisation strategy for patients $<75$ years of age with cardiogenic shock. ${ }^{3}$ In this article, we review the incidence, aetiology, prevention, and recognition of shock, as well as its management.

\title{
INCIDENCE
}

The extent of myocardial salvage from reperfusion treatment decreases exponentially with time to re-establishing coronary flow. Unfortunately, there has been little progress in reducing time to hospital presentation over the past decade, ${ }^{4}$ and this perhaps accounts for the stagnant incidence of cardiogenic shock in community studies $(7.1 \%) .{ }^{5}$ Cardiogenic shock also complicates non-ST elevation acute coronary syndromes. The incidence of shock in the PURSUIT trial was $2.9 \%$ (1995-97), ${ }^{6}$ similar to the $2.5 \%$ incidence reported in the non-ST elevation arm of the GUSTO II-B trial (1994-95). ${ }^{7}$ A number of strategies that centre on reducing the time to effective treatment may help decrease the incidence of shock. These include public education to decrease the time to hospital presentation, triage and early transfer of high risk patients to selected centres, and early primary percutaneous coronary intervention (PCI) or rescue PCI for failed thrombolysis in high risk patients.

\section{PREDICTING AND PREVENTING SHOCK}

The onset of cardiogenic shock in a patient following ST elevation MI heralds a dismal in-hospital prognosis. The $7.2 \%$ of patients developing shock in the GUSTO-I trial accounted for $58 \%$ of the overall deaths at 30 days. ${ }^{8}$ Similarly, the 30 day death rates with non-ST elevation MI cardiogenic shock in the PURSUIT and GUSTO-II b databases were $66 \%$ and $73 \%$, respectively. Even with early revascularisation, almost $50 \%$ die at 30 days. The prevention of shock is therefore the most effective management strategy. The opportunity for prevention is substantial, given the observation that only a minority of patients (10-15\%) present to the hospital in cardiogenic shock. Whether due to pump failure or a mechanical cause, shock is predominantly an early in-hospital complication in the ST elevation MI setting. The median time post-MI for occurrence of shock in the randomised SHOCK trial was 5.0 (interquartile range 2.2-12) hours. Similarly, median time from MI onset to development of shock in the SHOCK registry was 6.0 (1.8-22.0) hours, and median time from hospital admission was 4 hours. Shock complicating unstable angina/non-Q MI occurs at a later time period. In the GUSTO-IIb trial shock was recognised at a median of 76.2 (20.6-144.5) hours for non-ST elevation MI compared to 9.6 (1.8-67.3) hours with ST elevation MI ( $<<0.001)$, and median time to shock in the non-ST elevation PURSUIT trial was 94.0 (38-206) hours.

A primary goal in preventing shock should be an effort to reduce the large proportion of patients presenting with acute ST elevation MI who do not receive timely reperfusion treatment. Successful early reperfusion of the infarct related coronary artery while maintaining integrity of the downstream microvasculature limits ongoing necrosis, salvages myocardium, and may prevent the development of shock in many vulnerable patients. In-hospital development of shock often follows failed thrombolysis or successful thrombolysis followed by evidence of recurrent MI (ST re-elevation), infarct extension (ST elevation in new leads), and recurrent ischaemia (new ST depression). These complications may be significantly reduced by a primary PCI strategy. Currently, a minority of hospitals in the USA and an even smaller proportion worldwide possess the infrastructure and personnel to perform primary PCI effectively. 


\section{Trial acronyms}

DIGAMI: Diabetes mellitus Insulin Glucose infusion in Acute Myocardial Infarction

FTT: Fibrinolytic Therapy Trialists

GUSTO: Global Utilization of Streptokinase and Tissue

plasminogen activator for Occluded coronary arterie

PURSUIT: Platelet glycoprotein Ilb/Illa in Unstable angina:

Receptor Suppression Using Integrilin Therapy

SHOCK: SHould we emergently revascularize Occluded

Coronaries for cardiogenic shock ?

SMASH: Swiss Multicenter trial of Angioplasty for SHock

Recognising patients at highest risk for development of shock may facilitate the early transfer of high risk patients before onset of haemodynamic instability. Early referral of high risk patients for rescue angioplasty in the setting of thrombolytic failure may also prove beneficial.

A number of scoring systems using predictive models for the development of shock have been reported to aid with this decision strategy. In the GUSTO-I study, age, systolic blood pressure, heart rate, and presenting Killip class accounted for $>85 \%$ of the predictive information. The same four variables were significant in the GUSTO III population and accounted for $>95 \%$ of the predictive information, with a validated concordance index of $0.796 .{ }^{9}$ Major predictors of shock in the PURSUIT population included age, systolic blood pressure, ST depression on presenting ECG, heart rate, height, enrolling MI, and rales on physical examination. Although these scoring systems can be useful, the limitations of these databases need to be stressed. Patients enrolled in randomised clinical trials are themselves selected. Furthermore, positive predictive value for a patient with maximum attainable scores in the GUSTO-I and PURSUIT model are only 50\% and 35\%, respectively. ${ }^{10}$

\section{CLINICAL RECOGNITION}

Treatment cannot be initiated unless the clinical entity is recognised. Cardiogenic shock is characterised by inadequate tissue perfusion in the setting of adequate intravascular volume. Specifically, shock in the peri-infarction setting is defined as sustained hypotension (systolic blood pressure $\leqslant 90 \mathrm{~mm} \mathrm{Hg}$ for $\geqslant 30$ minutes), accompanied by signs of peripheral hypoperfusion (altered mental status, cool peripheries, oliguria). This clinical entity is unresponsive to fluid resuscitation alone, with a cardiac index $<2.2 \mathrm{l} / \mathrm{min} / \mathrm{m}^{2}$. Subjects requiring pharmacologic or mechanical circulatory support to maintain blood pressure are also included in this category. However, there is a wide spectrum of clinical symptoms, signs, and haemodynamic findings and variability in the severity of shock. It should be diagnosed in all patients exhibiting signs of inadequate tissue perfusion irrespective of blood pressure. Some patients, particularly those with anterior MI, develop signs of end organ hypoperfusion in the setting of unsupported blood pressure measurements $>90 \mathrm{~mm} \mathrm{Hg}$. The urine output is typically low and the heart rate $>90$ beats per minute. This "pre-shock" presentation is associated with a high risk of in-hospital morbidity and mortality (43\%). ${ }^{11}$ When the physician fails to recognise that the tachycardia is caused by a pronounced reduction in stroke volume and therefore administers $\beta$ blockers, frank shock may be precipitated.
In the SHOCK trial registry, $64 \%$ of patients presented typically with hypotension, evidence of ineffective cardiac output (resting tachycardia, altered mental status, oliguria, cool peripheries), and pulmonary congestion. ${ }^{12}$ A substantial minority (28\%) presented with evidence of hypoperfusion in the absence of pulmonary congestion-the "silent lung" syndrome. These latter patients have an equal distribution of anterior (50\%) and non-anterior index infarctions (50\%) with pulmonary capillary wedge pressure in the range of 21.5 $\pm 6.7 \mathrm{~mm} \mathrm{Hg}$. Inexperienced clinicians may inappropriately treat such patients with large fluid boluses akin to the management of hypotension with right ventricular infarction. ${ }^{13}{ }^{14}$ Unadjusted in-hospital mortality for this group in the SHOCK registry exceeded that for the classical presentation $(70 \% \quad v 60 \%, \mathrm{p}=0.036)$, a difference that was non-significant after adjustment. These data highlight the clinical importance of the subjective signs of hypoperfusion obtained on physical examination in this population. In the GUSTO-I mortality model, altered sensorium (odds of dying $1.68,95 \%$ confidence intervals (CI) 1.19 to 2.39 ), cold clammy skin (odds of dying $1.68,95 \%$ CI 1.15 to 2.46 ), and oliguria (odds of dying 2.25, 95\% CI 1.61 to 3.15 ) were associated with an increased 30 day mortality independent of haemodynamic variables..$^{15}$

\section{AETIOLOGY}

There are several possible causes of cardiogenic shock in the setting of MI-left ventricular dysfunction, right ventricular dysfunction, and mechanical complications (fig 1). Recognition of shock should immediately lead to a quest for its cause. A combination of the history, physical findings, ECG, and a screening echocardiogram (table 1) will enable the clinician to arrive quickly at an accurate diagnosis. A right heart catheterisation is often not necessary for diagnosis and need only be performed when there is continued doubt or to guide management when shock does not rapidly resolve. Predominant left ventricular pump failure in the setting of a large MI is the most common aetiology. Ventricular septal rupture, severe mitral regurgitation, cardiac rupture, and tamponade should be excluded and haemorrhagic shock considered, especially in the elderly. Although the typical findings of significant right ventricular infarction are hypotension, clear lung fields, and jugular venous distension, severe right ventricular dysfunction (with or without excess fluid administration) may result in left ventricular compromise caused by right ventricular distension and septal shift, resulting in clinical evidence of pulmonary congestion. Systolic anterior motion of the anterior mitral leaflet causing left ventricular outflow tract obstruction in the MI setting has also been reported. Other masqueraders in this situation include aortic dissection and massive pulmonary embolism, which should be considered in the appropriate clinical context. The latter includes discordance between extent of ECG and haemodynamic abnormalities - that is, mild to moderate ECG abnormalities in the setting of severe haemodynamic derangement.

\section{MANAGEMENT OF CARDIOGENIC SHOCK CAUSED BY PREDOMINANT LEFT VENTRICULAR FAILURE}

Reports of dramatic declines in mortality with early revascularisation for cardiogenic shock began to emerge in the late 1980s. ${ }^{16-18}$ Dedicated investigators in selected centres reported these single centre observations which were, however, prone to selection and publication bias. Randomised clinical trials testing the superiority and generalisability of an early 


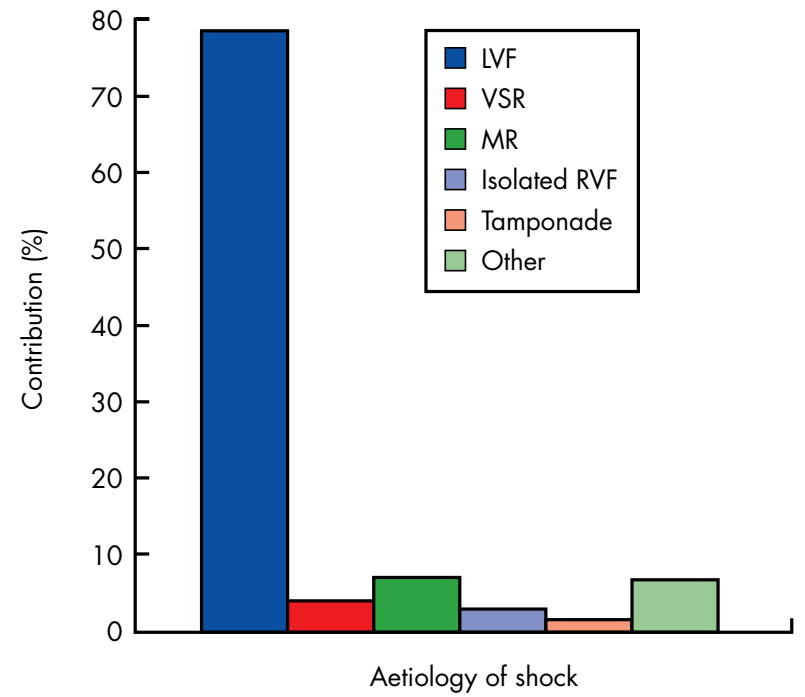

Figure 1 Aetiology of suspected cardiogenic shock in the combined SHOCK trial registry and trial (total $n=1422$, only first 232 trial patients are included). "Other" includes shock caused by prior severe valvar disease, dilated cardiomyopathy, excess $\beta$ blockade/calcium channel blockade, haemorrhage, and procedura complications. Aortic dissection, pulmonary embolism, and dynamic subaortic outflow obstruction should also be considered. LVF, left ventricular function; $M R$, mitral regurgitation; RVF, right ventricular failure; VSR, ventricular septal rupture.

Table 1 Usefulness of echocardiography in cardiogenic shock

- Evaluate left ventricular function and myocardium at risk

- Evaluate remote myocardial segments

- Screen for ventricular septal rupture

- Screen for severe mitral regurgitation and proceed to

transoesophageal echocardiography as needed

- Look for tamponade/rupture

- Assess right ventricular function

- Look for aortic dissection

revascularisation strategy were clearly warranted and the National Heart, Lung, and Blood Institute funded the SHOCK trial in the USA, while the SMASH trial in Switzerland evaluated the same issue. ${ }^{19}{ }^{20}$ While SMASH failed to recruit an adequate number of patients, SHOCK reported an increase in 30 day survival from $46.7 \%$ to $56.0 \%$ by the adoption of an early revascularisation strategy, but this absolute $9 \%$ difference did not reach significance $(p=0.11)$. On follow up, the survival difference in favour of the early revascularisation strategy became larger and significant at six months $(36.9 \% \mathrm{v}$ $49.7 \%, \mathrm{p}=0.027)$ and one year $(33.6 \% v 46.7 \%)$ for an absolute reduction of $13.2 \%$ (95\% CI $2.2 \%$ to $24.1 \%$, p < 0.03 ). The Kaplan-Meier survival curves for the early revascularisation and initial medical stabilisation arms are illustrated in fig 2 . There were 10 prespecified subgroup variables examined, including sex, age, prior MI, hypertension, diabetes, anterior MI, early or late shock, and transfer or direct admission status. A benefit of early revascularisation was demonstrated for all subgroups except for the elderly. Age $\geqslant 75$ versus $<75$ years interacted significantly with treatment effect at 30 days, six months, and one year. The benefit of early revascularisation was large for those $<75$ years at 30 days (41.4\% v 56.8\%, 95\% CI $-27.8 \%$ to $-3.0 \%$ ), and six months ( $44.9 \% \vee 65.0 \%, 95 \%$ CI

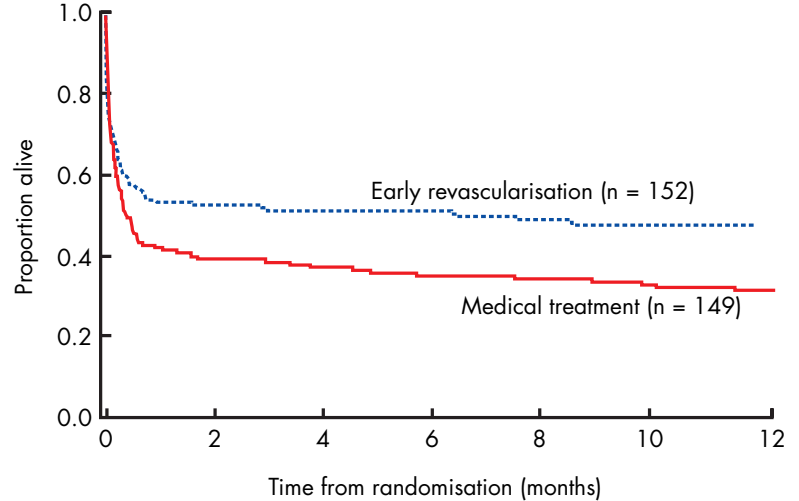

Figure 2 Kaplan-Meier curve showing 12 month survival in the early revascularisation and initial medical stabilisation arms of the SHOCK trial. Reproduced from Hochman et al, ${ }^{2}$ with permission of the American Medical Association.

$-31.6 \%$ to $-7.1 \%$ ) and was not apparent for the elderly (see below). An increased utilisation of revascularisation was also associated with improved outcome in the GUSTO-I thrombolytic trial and favourable outcomes in recent registries. ${ }^{21}{ }^{22} \mathrm{An}$ algorithm for the management of cardiogenic shock is outlined in fig 3.

\section{Step 1: immediate resuscitation measures}

The goal is to prevent devastating end organ injury while the patient is being transported for definitive treatment. Maintenance of adequate mean arterial pressure to prevent adverse neurologic and renal sequelae is vital. Dopamine or noradrenaline (norepinephrine), depending on the degree of hypotension, should be initiated promptly to raise mean arterial pressure and be maintained at the minimum dose required. Dobutamine may be combined with dopamine at moderate doses or used alone for a low output state without frank hypotension. Intra-aortic balloon counterpulsation should be initiated before transportation when facilities are available. Arterial blood gas and oxygen saturation should be monitored with early institution of continuous positive airway pressure or mechanical ventilation as needed. The ECG should be monitored continuously, and defibrillating equipment, intravenous amiodarone, and lidocaine should be readily available. (Thirty three per cent of patients in the early revascularisation arm of the SHOCK trial had cardiopulmonary resuscitation, sustained ventricular tachycardia or ventricular fibrillation before randomisation.) Transcutaneous pacing electrodes as well as provisions for temporary transvenous pacing should be placed at the patient's bedside. Aspirin and full dose heparin should be administered. For ST elevation MI requiring transfer for angiography, we recommend intra-aortic balloon pump (IABP) placement at the local hospital when possible. A fibrinolytic agent should be initiated in patients with ST elevation MI if the anticipated delay to angiography is more than two hours. Thirty five day mortality for patients with systolic blood pressure $<100 \mathrm{~mm} \mathrm{Hg}$ receiving thrombolysis in the FTT meta-analysis was $28.9 \%$ compared to $35.1 \%$ with placebo. This translates into 62 lives saved ( $95 \%$ CI 26 to 98, p < 0.001 ) per 1000 patients treated. ${ }^{23}$ Augmentation of blood pressure with an IABP in this situation may facilitate thrombolysis by increasing coronary perfusion pressure. Similarly, raising blood pressure (to $130 \mathrm{~mm} \mathrm{Hg}$ systole) by using vasopressor support has also shown synergism in experimental models, but this increase is difficult to achieve in patients in shock. For 


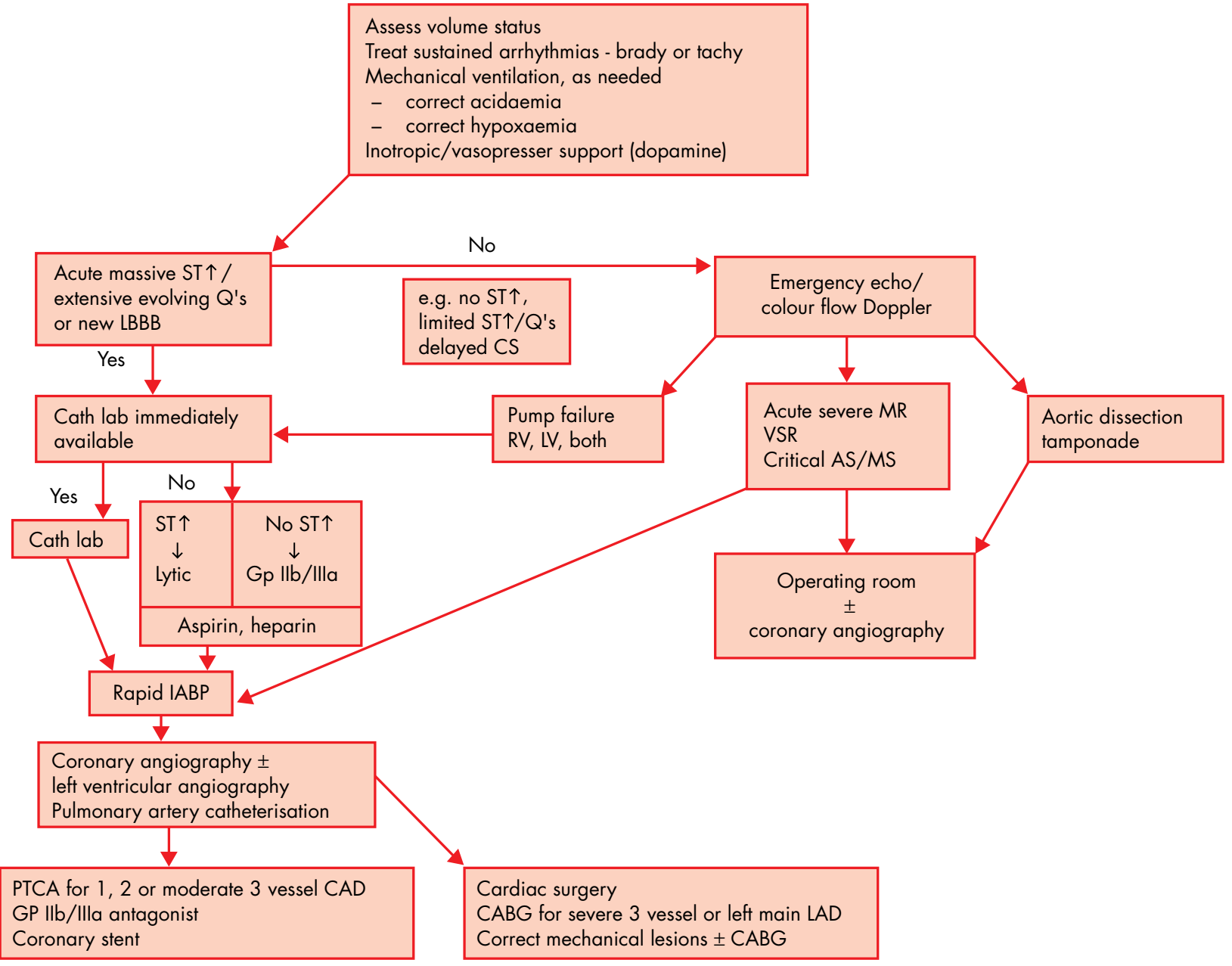

Figure 3 Algorithm on management of cardiogenic shock following ST elevation myocardial infarction. AS, atrial stenosis; CABG, coronary artery bypass graft; $C A D$, coronary artery disease; IABP, intra-aortic balloon pump; $L A D$, left anterior descending; $L B B B$, left bundle branch block; LV, left ventricle; MR mitral regurgitation; MS, mitral stenosis; PTCA, percutaneous transluminol coronary angioplasty; RV, right ventricle; VSR, ventricular septal rupture. Reproduced from Topol EJ (ed) Textbook of cardiovascular medicine, 2nd ed, with permission.

non-ST elevation MI cardiogenic shock awaiting catheterisation, a glycoprotein IIb/IIIa inhibitor should be initiated.

\section{Step 2: early definition of coronary anatomy}

This is the pivotal step in the management of cardiogenic shock resulting from predominant ischaemic pump failure. Patients in a community hospital setting should be emergently transferred/airlifted to an experienced designated regional tertiary care facility. The referring and accepting physician as well as the critical care transport team should be in constant communication to avoid delays in cardiac catheterisation. Prophylactic IABP placement is recommended before transfer and otherwise before angiography; radiocontrast use should be minimised. Early reversal of hypotension with IABP support serves as an excellent prognostic marker for survival, but those who do or do not respond well to IABP both derive benefit from early revascularisation. If a high quality echocardiogram has already been performed, a ventriculogram need not be repeated. Shock is characterised by a high incidence of triple vessel disease, left main disease, and impaired left ventricular function. ${ }^{24}$ The mean (SD) left ventricular ejection fraction for patients in the SHOCK trial and registry was 29 (11)\% and $34(14) \%$, respectively. The extent of ventricular dysfunction and haemodynamic instability should be correlated with coronary anatomy. An isolated circumflex lesion or a right coronary lesion should rarely manifest as shock in the absence of right ventricular infarction, left ventricular underfilling, bradyarrhythmia or prior MI or cardiomyopathy. In situations like this it is important for the clinician to immediately consider and exclude mechanical and other aetiologies of cardiogenic shock.

\section{Step 3: perform early revascularisation}

Definition of anatomy should be followed rapidly by selection of the modality of revascularisation. PCI will most often be the treatment of choice. Glycoprotein IIb/IIIa antagonists and stenting of the infarct related artery are indicated, although trial data are lacking. Recent reports suggest an additive benefit of stenting and glycoprotein IIb/IIIa antagonists in cardiogenic shock similar to the remainder of the clinical spectrum of PCI. ${ }^{25}$ However, if there is sluggish flow despite absence of post-coronary angioplasty stenosis, we recommend waiting until flow normalises before stenting. Stenting may exacerbate distal embolisation. Glycoprotein IIb/IIIa antagonists may improve reflow. Intracoronary adenosine or nitroprusside may be tried. There is no randomised clinical evidence to support multivessel angioplasty in this setting, and the decision to perform angioplasty in the non-infarct related artery should be individualised. In selected cases, with remote ischaemia, 


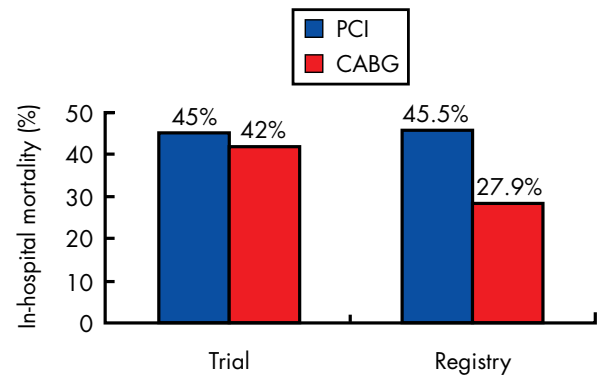

Figure 4 In-hospital mortality with percutaneous coronary intervention (PCI) and coronary artery bypass graft surgery (CABG) in the early revascularisation arm of the randomised SHOCK trial compared to the non-randomised larger SHOCK registry.

non-infarct related artery critical stenosis, and lack of haemodynamic improvement after infarct related artery PCI (with IABP support), revascularisation of the non-infarct territory may play a role. In patients with moderate three vessel disease, emergent PCI of the infarct related artery with consideration for later coronary artery bypass graft surgery (CABG) is preferred based on the concern that distal embolisation in non-infarct related artery segments is not tolerated in shock.

There are no trials randomising patients to PCI versus CABG in the setting of cardiogenic shock. The safety and feasibility of CABG in this situation is well documented. Severe triple vessel and left main coronary artery disease with severely impaired left ventricular function predominate in the shock setting. Emergent CABG allows the opportunity to achieve complete revascularisation and rectify severe mitral regurgitation while cardiopulmonary bypass maintains systemic perfusion. The SHOCK trial protocol recommended emergency CABG for patients with left main or severe three vessel disease. The in-hospital mortality rates with CABG in the SHOCK trial and registry were the same as the outcomes with PCI despite more severe coronary artery disease and twice the rate of diabetes in patients who underwent CABG (fig 4). We believe that CABG is underused in the shock setting. When dictated by anatomy, we recommend emergent CABG with pre-induction IABP support. The potential for benefit with metabolic support in this situation is large but remains formally untested in the shock setting.

Intra-aortic balloon counterpulsation support

Consistent with the current ACC/AHA guidelines, we recommend early consideration of IABP placement for patients with cardiogenic shock who are candidates for an aggressive strategy. Although randomised controlled trial data are lacking, benefit is seen across a number of observational databases. ${ }^{26-29}$ It provides excellent temporary haemodynamic support in many patients. ${ }^{30}$ It must also be noted that in the randomised SHOCK trial use of IABP was strongly recommended in both the early revascularisation and conservative arm. IABP utilisation was $87 \%$ in this trial and may have contributed to the improved outcomes observed in both groups compared to historical controls. The observed rates of IABP utilisation in US sites increased from 35\% in GUSTO-I to 47\% in GUSTO-III $(\mathrm{p}=0.001) .{ }^{31}$ In contrast, utilisation at non-US sites in both trials were low ( $7 \%$ and $10 \%$, respectively). We believe that IABP is currently underutilised in the setting of shock and strongly recommend that community hospitals attempt to develop an IABP programme so that treatment may be initiated before transfer whenever possible.

\section{ISSUES IN SHOCK MANAGEMENT} How should the elderly be treated?

Although there was no apparent benefit from an early revascularisation strategy in patients aged $\geqslant 75$ years in the SHOCK trial, the total number of patients in this subgroup was small $(n=56)$. The $17 \%$ of 277 patients aged $>75$ years in the concomitant SHOCK registry who were selected for early revascularisation appeared to derive benefit that was similar to their younger counterparts, even after covariate adjustment. ${ }^{32}$ We do not feel there is adequate evidence to categorically deny early revascularisation to the elderly. We use an individualised approach to the elderly. A select group based on prior functional status, "physiologic" age, comorbidity, proximity of MI, duration of shock, and coronary anatomy may be offered an early revascularisation strategy. In the absence of contraindications, the remainder are treated with thrombolysis with or without IABP, or comfort care alone for those unlikely to benefit (see below). The very elderly often request comfort care alone.

\section{Is there a therapeutic window for emergency revascularisation?}

Early revascularisation should be considered as soon as possible following diagnosis of cardiogenic shock. The median time from randomisation to revascularisation was $1.4(0.6-2.8)$ hours and the median time from MI to randomisation was 11 hours in the randomised SHOCK trial. However, it should be noted that patients were eligible for the trial if shock was diagnosed within 36 hours of index MI and randomisation performed within 12 hours of shock onset. There was no significant interaction between time from MI to randomisation and treatment effect. Ongoing ischaemia and stuttering necrosis are typical in the vicious cycle of ischaemiahypoperfusion that characterise shock. Unlike primary reperfusion treatment, the window of opportunity in this setting is large. We recommend an early revascularisation strategy for shock patients up to 48 hours post-index MI and up to 18 hours post-shock. Patients who are not revascularised within 18 hours of shock onset but survive the early phase with resolution of shock should undergo coronary angiography. Revascularisation should be performed based on standard post-MI criteria-that is, triple vessel or left main disease or spontaneous or inducible ischaemia.

\section{What is the quality of life?}

Although the SHOCK trial showed that 13 lives were saved at one year per 100 patients treated with an early revascularisation strategy, it was important to document that survivors had an acceptable quality of life. It is reassuring that $83 \%$ of one year survivors $(\mathrm{n}=90)$ were in New York Heart Association heart failure functional class I or II at 12 months.

\section{Is care ever futile?}

It is difficult to assess futility in the acute critical setting that characterises cardiogenic shock. It is vital to establish communication with the family as soon as the clinical entity is recognised. The patient or surrogate should play a role in the decision making process. Patients who require prolonged cardiopulmonary resuscitation and sustain presumed anoxic brain damage and those with other life shortening illnesses are not candidates for aggressive care. Although cardiac index, blood pressure, signs of hypoperfusion, and ejection fraction are independently associated with outcome, the beneficial effect of early revascularisation was noted across the spectrum of subgroups, except the very elderly. Further research is 
required to develop a risk score to identify other patients with very poor outcomes despite early revascularisation. This may enable effective resource utilisation in the future and prevent heroic manoeuvres for patients unlikely to benefit.

\section{MANAGEMENT OF MECHANICAL COMPLICATIONS}

The utilisation of early reperfusion strategies has decreased the incidence of mechanical complications post ST-elevation MI. ${ }^{33}{ }^{34}$ Overall incidence of ventricular septal rupture in the GUSTO-I trial was $0.2 \%(84 / 41021)$ with a 30 day mortality of $73.8 \%{ }^{35}$ Although IABP may help achieve temporary haemodynamic stability, prognosis following onset of haemodynamic collapse is grim. $^{36}$ The overall in-hospital mortality for ventricular septal rupture complicated by cardiogenic shock in the SHOCK registry was $87 \%$ (47/55) with an $81 \%(25 / 31)$ surgical mortality. A significant number of patients will have ventricular septal rupture without early evidence of circulatory collapse. Onset of circulatory collapse in this situation is unpredictable, and a superior surgical outcome is realised when emergent surgery is performed before the onset of cardiogenic shock. In keeping with the ACC/AHA guidelines, we recommend urgent surgery for our patients with newly diagnosed ventricular septal rupture. Similarly, all patients with mechanical mitral regurgitation and subacute rupture should be emergently considered for surgical intervention.

\section{FUTURE DIRECTIONS}

The role of L-NMMA, a selective nitric oxide inhibitor, is promising in this setting. ${ }^{37}$ The utility of GIK (glucose, insulin, and potassium) metabolic support for cardiogenic shock patients is an intriguing but unanswered question. However, we recommend intensive insulin treatment to normalise blood glucose in those with elevated values. The DIGAMI study suggested that this strategy was beneficial for acute MI patients. In a study of intensive care unit patients, those with hyperglycaemia who were randomised to intensive insulin had reduced mortality rates. ${ }^{38}$

The role of selection of patients for wearable left ventricular assist devices and their clinical utility in the setting of shock following MI needs to be explored. Patients who are candidates for cardiac transplantation should receive bridging left ventricular assist devices.

\section{CONCLUSION}

Early recognition and transfer of high risk patients and adoption of a primary PCI strategy may decrease the incidence of cardiogenic shock. Establishing the aetiology of shock and early definition of coronary anatomy in the setting of pump failure are crucial. We recommend urgent revascularisation supported by IABP for patients aged $<75$ years in cardiogenic shock caused by pump failure. A selective approach is advocated for the elderly. Regional care centres that are experienced in the management of shock should be designated and protocols developed for rapid transport of critically ill patients. Further research is needed in the areas of pharmacologic and mechanical haemodynamic support, refinement of revascularisation strategies, and outcome modelling.

\section{Authors' affiliations}

V Menon, Division of Cardiology, University of North Carolina, Chapel Hill, North Carolina, USA

J S Hochman, Division of Cardiology, St Luke's-Roosevelt Hospital Center, Columbia College of Physicians \& Surgeons, New York, USA

\section{REFERENCES}

1 Hochman JS, Sleeper LA, Webb JG, et al. Early revascularization in acute myocardial infarction complicated by cardiogenic shock. N Engl J Med 1999;341:625-34

- Randomised controlled trial comparing an early revascularisation strategy to an initial medical stabilisation strategy in the setting of cardiogenic shock.

2 Hochman JS, Sleeper LA, White HD, et al. One-year survival following early revascularization for cardiogenic shock. JAMA 2001;285:190-2.

One year follow up of the SHOCK trial.

3 Ryan TJ, Anderson JL, Antman EM, et al. 1999 update: ACC/AHA guidelines for the management of patients with acute myocardial infarction. A report of the American College of Cardiology/American Heart Association task force on practice guidelines. (Committee on management of acute myocardial infarction). Circulation 1999;100:1016-30.

4 Rogers WJ, Canto JG, Lambrew CT, et al. Temporal trends in the treatment of 1.5 million patients with myocardial infarction in the US from 1990 through 1999. J Am Coll Cardiol 2000;36:2056-63.

5 Goldberg RJ, Samad NA, Yarzebski J, et al. Temporal trends in cardiogenic shock complicating acute myocardial infarction. N Engl J Med 1999;340: 1162-68

- Large community study showing a decline in mortality but no change in incidence of shock over time.

6 Hasdai D, Harrington RA, Hochman JS, et al. Platelet glycoprotein Ilb/IIla blockade and outcome of cardiogenic shock complicating acute coronary syndromes without persistent ST- segment elevation. J Am Coll Cardiol 2000;36:685-92

7 Holmes DR Jr, Berger PB, Hochman JS, et al. Cardiogenic shock in patients with acute ischemic syndromes with and without ST-segment elevation. Circulation 1999;100:2067-73.

8 Holmes DR Jr, Bates ER, Kleiman NS, et al. Contemporary reperfusion therapy for cardiogenic shock: the GUSTO-I trial experience. J Am Coll Cardiol 1995;26:668-74.

9 Hasdai D, Califf RM, Thompson TD, et al. Predictors of cardiogenic shock after thrombolytic therapy for acute myocardial infarction. J Am Coll Cardiol 2000;35:136-43.

10 Hasdai D, Topol EJ, Califf RM, et al. Cardiogenic shock complicating acute coronary syndromes. Lancet 2000;356:749-56.

- Recent review with emphasis on predictive modelling.

11 Menon V, Slater JN, White HD, et al. Acute myocardial infarction complicated by systemic hypoperfusion without hypotension: report from the SHOCK trial registry. Am J Med 2000;108:374-80.

- Clinical description and outcome of "pre-shock".

12 Menon V, White H, Lejemtel T, et al. The clinical profile of patients with suspected cardiogenic shock due to predominant left ventricular failure. A report from the SHOCK trial registry. J Am Coll Cardiol 2000;36:1071-6.

13 Swan HJ, Forrester JS, Diamond G, et al. Hemodynamic spectrum of myocardial infarction and cardiogenic shock; a conceptual model. Circulation 1972;45:1097-110

14 Forrester JS, Diamond GA, Swan HJC. Correlative classification of clinical and hemodynamic function after acute myocardial infarction. Am J Cardiol 1977;39:137-45.

15 Hasdai D, Holmes DR Jr, Califf RM, et al. Cardiogenic shock complicating acute myocardial infarction: predictors of death. Am Heart J 1999;138:21-31

16 Lee L, Bates ER, Pitt B, et al. Percutaneous transluminal coronary angioplasty improves survival in acute myocardial infarction complicated by cardiogenic shock. Circulation 1988;78:1345-51.

17 Verna E, Repetto S, Boscarini $M$, et al. Emergency coronary angioplasty in patients with severe left ventricular dysfunction or cardiogenic shock after acute myocardial infarction. Eur Heart J 1989; 10:958-66.

18 Moosvi AR, Khaja F, Villanueva L, et al. Early revascularization improves survival in cardiogenic shock complicating myocardial infarction. J Am Coll Cardiol 1992;19:907-14.

19 Hochman JS, Sleeper LA, Godfrey E, et al. Should we emergently revascularize occluded coronaries for cardiogenic shock: an international randomized trial of emergency PTCA/CABG-trial design. Am Heart J 1999;137:313-21

20 Urban P, Stauffer JC, Bleed D, et al. A randomized evaluation of early revascularization to treat shock complicating acute myocardial infarction: the (Swiss) multicenter trial of angioplasty for shock-(S)MASH. Eur Heart J 1999:20:1030-8.

21 Berger PB, Holmes DR Jr, Stebbins L, et al. Impact of an aggressive invasive catheterization and revascularization strategy on mortality in patients with cardiogenic shock in the global utilization of streptokinase and tissue plasminogen activator for occluded coronary arteries (GUSTO-1) trial: an observational study. Circulation 1997;96:122-7.

22 Carnendran L, Abboud R, Sleeper LA, et al. Trends in cardiogenic shock: report from the SHOCK study. Eur Heart J 2001;22:472-8.

23 Fibrinolytic Therapy Trialists (FTT) Collaborative Group. Indications for fibrinolytic therapy in suspected acute myocardial infarction: collaborative overview of early mortality and major morbidity results from all randomized trials of more than 1000 patients. Lancet 1994;343:311-22.

24 Wong SC, Sanborn T, Sleeper LA, et al. Angiographic findings and clinical correlates in patients with cardiogenic shock complicating acute myocardial infarction: a report from the SHOCK trial registry. J Am Coll Cardiol 2000;36: 1077-83. 
25 Ajani AE, Maruff $P$, Warren R, et al. Impact of early percutaneous coronary intervention on short- and long-term outcomes in patients with cardiogenic shock after acute myocardial infarction. Am J Cardiol 2001;87:633-5

26 Waksman R, Weiss AT, Gotsman MS, et al. Intra-aortic balloon counterpulsation improves survival in cardiogenic shock complicating acute myocardial infarction. Eur Heart J 1993;14:71-4.

27 Kovack PJ, Rasak MA, Bates ER, et al. Thrombolysis plus aortic counterpulsation: improved survival in patients who present to community hospitals with cardiogenic shock. J Am Coll Cardiol 1997;29:1454-8.

28 Hudson MP, Granger CB, Stebbins A, et al. Cardiogenic shock survival and use of intraortic balloon counterpulsation: results from GUSTO-I and III trials. Circulation 1999;100:1-370.

29 Barron HV, Every N, Parsons LS, et al. The use of intra-aortic balloon counterpulsation in patients with cardiogenic shock complicating acute myocardial infarction; data from the National Registry of Myocardial Infarction 2. Am Heart J 2001;141:933-9

30 Scheidt S, Wilner G, Mueller H, et al. Intra-aortic balloon counterpulsation in cardiogenic shock: report of a cooperative clinical trial. N Engl J Med 1973;288:979-84.

- Classic early paper on the utility of IABP to stabilise patients in the setting of shock.

31 Menon V, Hochman JS, Stebbins A, et al. Lack of progress in cardiogenic shock: lessons from the GUSTO trials. Eur Heart J 2000;21:1928-36.
32 Dzavik V, Sleeper LA, Saucedo J, et al. Early revascularization is associated with improved survival in patients aged $\geqslant 75$ years with acute myocardial infarction complicated by cardiogenic shock: a report from the SHOCK registry. J Am Coll Cardiol 2002;39:330A.

33 Gertz SD, Kragel AH, Kalan JM, et al. Comparison of coronary and myocardial morphologic findings in patients with and without thrombolytic during fatal first myocardial infarction. Am J Cardiol 1990;66:904-9.

34 Honan MB, Harrell FE Jr, Reimer KA, et al. Cardiac rupture, mortality and timing of thrombolytic therapy: a meta-analysis. J Am Coll Cardiol 1990;16:359-67.

35 Crenshaw BS, Granger CB, Birnbaum Y, et al. Risk factors, angiographic patterns, and outcomes in patients with ventricular septal defect complicating acute myocardial infarction. Circulation 2000;101:27-32.

36 Menon V, Webb JG, Hillis LD, et al. Outcome and profile of ventricular septal rupture with cardiogenic shock after myocardial infarction: a report from the SHOCK trial registry. J Am Coll Cardiol 2000;36:1110-6.

- Largest clinical experience with ventricular septal rupture in the setting of cardiogenic shock.

37 Cotter G, Kaluski E, Blatt A, et al. L-NMMA la nitric oxide synthase inhibitor) is effective in the treatment of cardiogenic shock. Circulation 2000; 101:1358-61.

38 Van den Berghe G, Wouters P, Weekers F, et al. Intensive insulin therapy in critically ill patients. N Engl J Med 2001;345:1359-67. 